UCRL-JC-128034

PREPRINT

\title{
A Scaling Model for Laser-Produced Bubbles in Soft Tissue
}

\author{
R. A. London, D. S. Bailey, P. Amendt, \\ S. Visuri, and V. Esch
}

This paper was prepared for and presented at the SPIE International Symposium on BiOS ' 98 International Biomedical Optics Symposium San Jose, California

January 24-30, 1998

March 12, 1998

This is a preprint of a paper intended for publication in a journal or proceedings. Since changes may be made before publication, this preprint is made available with the understanding that it will not be cited or reproduced without the permission of the author. 


\section{DISCLAIMER}

This document was prepared as an account of work sponsored by an agency of the United States Government. Neither the United States Government nor the University of California nor any of their employees, makes any warranty, express or implied, or assumes any legal liability or responsibility for the accuracy, completeness, or usefulness of any information, apparatus, product, or process disclosed, or represents that its use would not infringe privately owned rights. Reference herein to any specific commercial product, process, or service by trade name, trademark, manufacturer, or otherwise, does not necessarily constitute or imply its endorsement, recommendation, or favoring by the United States Government or the University of California. The views and opinions of authors expressed herein do not necessarily state or reflect those of the United States Government or the University of California, and shall not be used for advertising or product endorsement purposes. 


\title{
A Scaling Model for Laser-Produced Bubbles in Soft Tissue
}

\author{
R. A. Londona, D. S. Baileya, P. Amendta, S. Visuria and V. Esch ${ }^{b}$ \\ a Lawrence Livermore National Laboratory, Livermore CA 94550 \\ b Endovasix, Inc., Belmont CA 94004
}

\begin{abstract}
The generation of vapor-driven bubbles is common in many emerging laser-medical therapies involving soft tissues. To successfully apply such bubbles to processes such as tissue break-up and removal, it is critical to understand their physical characteristics. To complement previous experimental and computational studies, an analytic mathematical model for bubble creation and evolution is presented. In this model, the bubble is assumed to be spherically symmetric, and the laser pulse length is taken to be either very short or very long compared to the bubble expansion timescale. The model is based on the Rayleigh cavitation bubble model. In this description, the exterior medium is assumed to be an infinite incompressible fluid, while the bubble interior consists of a mixed liquid-gas medium which is initially heated by the laser. The heated interior provides the driving pressure which expands the bubble. The interior region is assumed to be adiabatic and is described by the standard water equation-of-state, available in either tabular, or analytic forms. Specifically, we use adiabats from the equation-of-state to describe the evolution of the interior pressure with bubble volume. Analytic scaling laws are presented for the maximum size, the duration, and the energy of bubbles as functions of the laser energy and initially heated volume. Of particular interest, is the efficiency of converting laser energy into bubble motion.
\end{abstract}

\section{INTRODUCTION}

Laser produced vapor bubbles are important in many fields of laser medicine. They occur in cardiology in the applications of thrombolysis and angioplasty, in ophthalmology in the study of damage threshold, and in urology in lithotripsy, to mention a few applications. The effects of the vapor bubble can be either desired-as in the case of emulsifying clots in thrombolysis or undesired as in the case of damage to the eye.

Recently, much theoretical and experimental work has been done on elucidating the mechanisms of bubble formation, the dynamics of bubbles, and the effects on ambient soft tissue. In this paper we present a scaling model with the main goal of calculating the maximum bubble size for various input parameter values. The bubble size is of interest, as characteristic parameter of a bubble which indicates how much tissue it effects. In some cases it may be assumed that the maximum size of the bubble directly correlates with the amount of tissue which is broken up, or removed by the action of the bubble. We equate the maximum volume of the bubble to the removed tissue volume. This is not always the case, however the maximum bubble size remains an important parameter by which to characterize a laser produced bubble.

Our goal is to develop a simple and computationally quick model which enables estimates of the maximum bubble size and bubble duration. This then allows us both to understand the bubble dynamics on a fundamental level and to perform quick surveys of parameter space for the purpose of designing systems for various applications. The scaling model which we have developed to attain this goal is intermediate in complexity compared to various theoretical models which has been discussed in the literature.

The simplest model in the literature is the Rayleigh cavitation bubble model ${ }^{1}$. This model describes the expansion and collapse of an empty bubble in an ideal incompressible 
liquid, given initial conditions. The result often used is the formula for the bubble collapse expansion time (equal to the collapse time):

$$
t_{m} \approx \sqrt{\rho_{\text {ext }}} / P_{\text {ext }} R_{m}
$$

where $t_{m}$ is the expansion time (the m-subscript denotes the maximum bubble size expanding bubble), $R_{m}$ is the maximum radius, $\rho_{\text {ext }}$ and $P_{\text {ext }}$ are the density and pressure in the medium external to the bubble. In the Rayleigh model there is no gas and therefore no pressurc inside the bubble.

Our analysis extends the Rayleigh model by considering the gas inside the bubble-and the pressure which it exerts on the external medium. By using a self consistent description of the creation of this gas by vaporization of liquid by the laser, and by using an accurate equation-of-state (EOS) to describe the evolution of the energy and pressure of the internal gas, we have constructed a model which allows a calculation of the bubble dynamics. In particular we can calculate the maxim bubble size and the time from the deposited laser energy, $\left(Q_{L}\right)$, the external medium parameters in limiting cases of short and long laser pulse length $\left(t_{L}\right)$.

Many other models have been presented which include internal pressure and other effects in the Rayleigh model. Several of these are the inclusion of acoustic emission by Kirkwood and Bethe, and Plesset 2 , and the recent inclusion of an improved treatment of acoustic emission and material strength and failure by Glinsky et $\mathrm{al}^{3}$.

The most comprehensive models are the computational hydrodynamic simulations done by the Livermore and Los Alamos groups in the last few years 4 . These models include accurate EOSs, acoustic and shock wave emission, and non-spherical effects, and most recently, material strength effects. These models serve both to verify simpler models, as well as to compare to and guide experiments. They also allow the study of complicated 2-D effects such as vorticity generation and the creation of jets on bubble collapse.

\section{ELEMENTS OF BUBBLE SCALING MODEL}

\subsection{Assumptions.}

Several basic assumptions are made in deriving the scaling model. The geometry is assumed to be 1-dimensional with spherical symmetry. The correspondence between a realistic geometry associated with optical fiber delivery is illustrated in Figure 1. The essence of the approximation is to equate the initial volume of the spherical bubble with the volume into which the laser energy is deposited by the fiber. We also assume that the bubble is created by vaporization of the water, rather than cavitation. This requires a minimum laser energy necessary to bring the heated volume to a temperature above the boiling point of the water.

Another approximation is the neglect of heat conduction. We estimate that this is valid for heated volumes such that the heat conduction time $\left(R^{2} / 4 \alpha\right.$, where $a$ is the thermal diffusity) is greater than the Rayleigh time [Eq. (1)]. For properties of water, this occurs for $R_{i}>.06 \mu \mathrm{m}$, which include all cases of practical interest. We also ignore acoustic emission, which is valid for moderate laser energy densities $\left(Q_{L}<3 \mathrm{~kJ} / \mathrm{g}\right)$, but which breaks down for very high laser energy densities 5 . The model is based on two fundamental energy balance conditions, the first law of thermodynamics applied to the gas inside the bubble:

$$
d Q=d E-P d V
$$

and the balance of the kinetic energy acquired by the external fluid with the net work done by the bubble

$$
K_{e x t}=W \equiv \int\left(P-P_{e x t}\right) d V
$$


Here $Q$ is the enthalpy (heat) per unit mass, $E$ the internal energy, $P$ the pressure and $V$ the specific volume, both of the bubble interior, $K_{\text {ext }}$ the kinetic energy of the external fluid, and $W$ the net work done on the external fluid by the bubble. In addition we use an accurate EOS relating the pressure and energy of the internal gas to the temperature and density. In this paper we use a water EOS based on the NIST steam tables ${ }^{6}$. This is expected to accurately represent soft tissue, since water is the dominant constituent $(75 \%)$. The main effect of the tissue is to provide a shear strength which inhibits the growth of the bubble. We include an enhanced external pressure equal to that of the failure stress of the material. This prescription has been shown to be a valid, but approximate way to model the strength of the tissue (Glinsky et al). For typical soft tissues this value ranges from 1-10 bar.

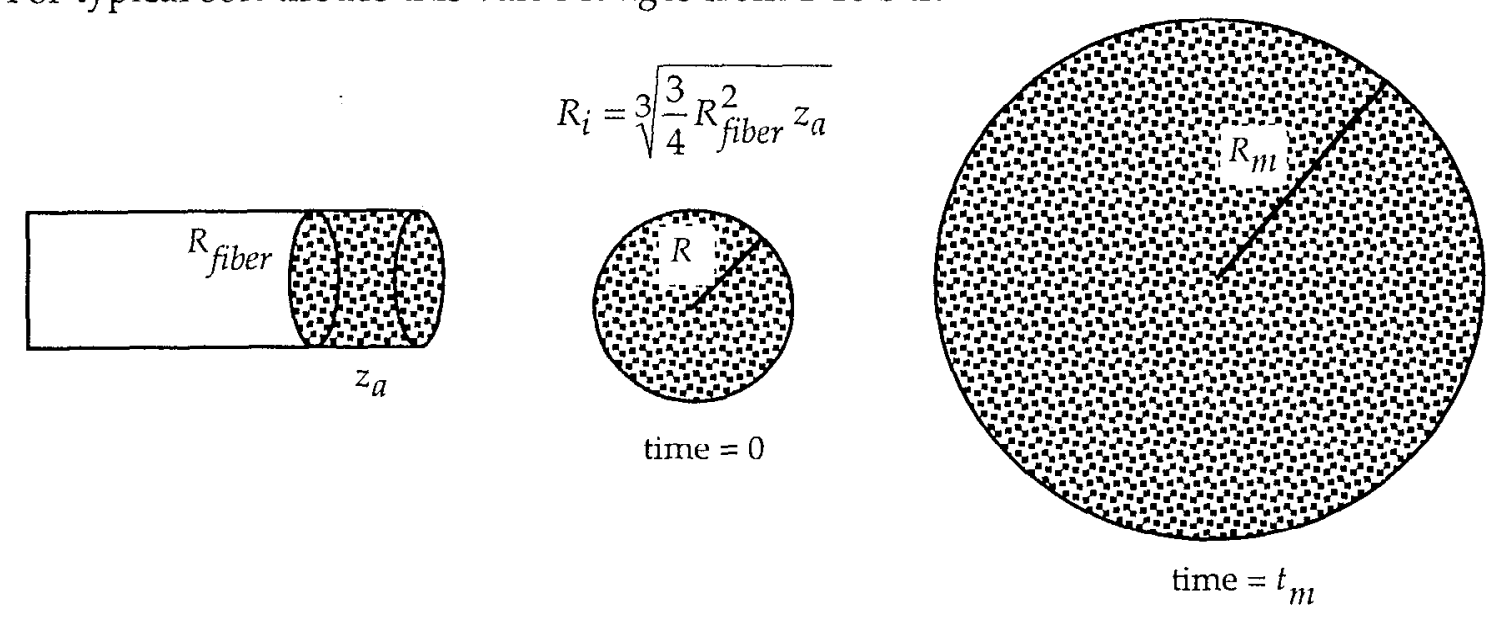

Figure 1. The deposition region for a fiber is approximated by a sphere of equal volume. $R_{\text {fiber }}$ is the fiber radius and $z_{\mathfrak{a}}$ the absorption length of the laser light in the fluid or tissue. The maximum bubble radius is $R_{m}$, reached at time $t_{m}$.

2.2 Qualitative Behavior for Long and Short Laser Pulses.

To calculate the bubble dynamics, we consider two limiting cases of the laser pulse length relative to a pressure equilibration timescale. Schematic illustrations of the bubble evolution are given in Figures $2 a$ and $b$ for the long and short pulse cases. In the case of long pulse, the pressure inside the bubble never greatly exceeds the internal pressure. The bubble grows in a near-equilibrium condition for the duration of the pulse. It reaches its maximum radius at the end of the laser pulse. Since the bubble does not overshoot its equilibrium radius as in the short pulse case, there is no real bubble collapse. The bubble may oscillate a bit about its equilibrium radius, and finally collapse when heat conduction cools the interior gas. In the short pulsc case the laser energy is deposited before the bubble can expand very much. The pressure builds up during the laser pulse, typically to a value much larger than the external pressure. The bubble radius begins to grow. As the internal pressure drops below the external pressure, the inertia of the external fluid allows the bubble to continue to grow. The bubble radius overshoots the equilibrium radius achieved in the long pulse case. At a time $t_{m}$ the bubble comes to rest at its maximum radius and then begins to collapse. The determination of the equilibrium time $\left(t_{e q}\right)$ is made self-consistently from the short pulse solution given below. 


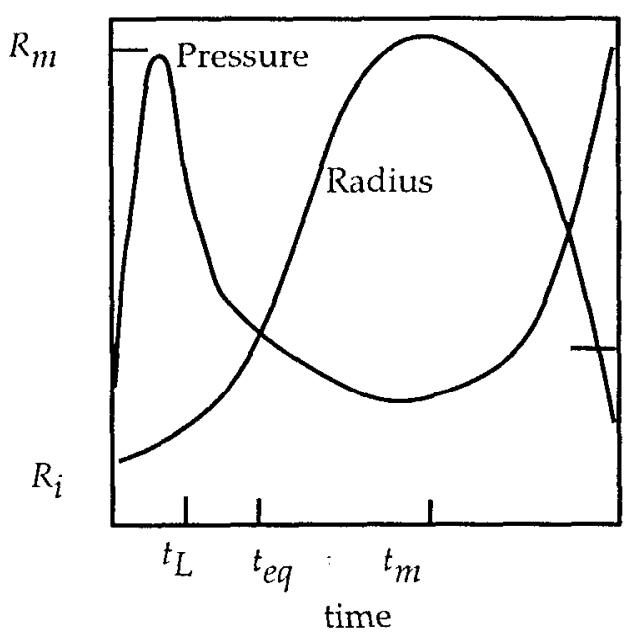

Figure 2a. Schematic representation of the time variation of the bubble radius and internal pressure for the short pulse case.

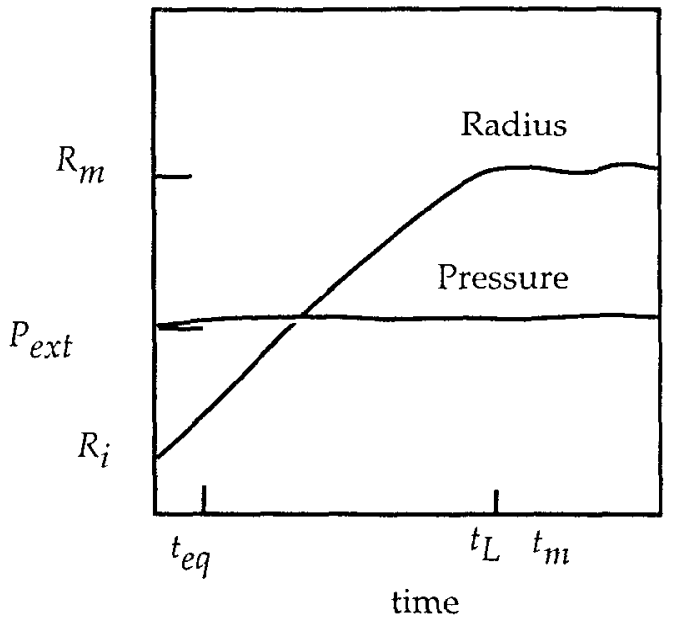

Figure $2 \mathrm{~b}$. Variation of the bubble radius and internal pressure for the long pulse case.

2.3 Long Pulse Limit: $t_{L}>>t_{e q}$.

For long pulses we find the maximum bubble size by assuming that the bubble expands at a pressure equal to the external pressure. We also assume that the temperature inside the bubble is equal to the equilibrium boiling temperature, which is $100^{\circ} \mathrm{C}$ for $P_{\text {ext }}=1 \mathrm{bar}$, but is somewhat larger for higher external pressure, e. g. $180^{\circ} \mathrm{C}$ for $P_{\text {ext }}=10$ bar. The later assumption can only hold when the specific laser energy is less than the latent heat at constant pressure. For higher laser energies the temperature will rise above the boiling point. The essence of this derivation is that the laser first heats the water to the boiling point and then the remainder of the energy goes into making water vapor at the boiling point. Given these assumptions, we can integrate Eq. (2) to give:

$$
\Delta Q_{L}=E_{m}-E_{o}+P_{e x t}\left(V_{m}-V_{o}\right)
$$

where the subscript " $\mathrm{o}$ " indicates conditions before the laser pulse. We use Eq. (4) in conjunction with the EOS, which is available as a FORTRAN program, to solve for $V_{O}$ given $Q_{L}$, $T_{O}$ (the initial temperature), and $P_{\text {ext }}$. Finally we find the maximum radius from the cube root of the volume. Results are presented in section III.

\subsection{Short Pulse Limit: $t_{L} \ll t_{e q}$.}

In the short pulse limit, we assume that the laser energy is deposited instantancouslybefore the bubble can expand. Then the expansion follows. Since no further heat is added, the expansion is adiabatic (or isentropic). Without solving for the time-dependent bubble dynamics, we can easily calculate the maximum bubble radius by finding the conditions when the kinetic energy is zero. From Eq. 3, this occurs when the net work is zero:

$$
\int\left(P-P_{\text {ext }}\right) d V=0
$$

We apply the first law (Eq. 2.) to the bubble in two steps. First it is used to equate the bubble internal energy immediately after the pulse to the initial internal energy plus the laser energy. Then it is applied in the adiabatic limit $(\mathrm{dQ}=0)$ after the laser pulse up to the time of maximum expansion. The net result is an expression for the first term in the integral in Eq. (5):

$$
\int P d V=\left(E_{o}+Q_{L}\right)-E_{m}
$$


The 2 nd term in the integral is straightforward since $P_{\text {ext }}=$ constant. We express the result of the evaluation of Eq. (5):

$$
E_{m}\left(V_{m l}\right)=\left(E_{o}+Q_{L}\right)+P_{e x t}\left(V_{m}-V_{o}\right) .
$$

Equation (7) is used along with the EOS, and the adiabatic condition for $P(V)$, to implicitly solve for the maximum volume and then the radius. The solution is accomplished by a simple numerical searching method. A graphical picture of the determination of the maximum volume is shown in Figure 3. Since we are considering only the period of bubble growth, the specific volume parameterizes time. We see the monotonic drop in bubble pressure with time. At first the kinetic energy grows in time. This is called the acceleration phase of the bubble dynamics. When the pressure drops below the external pressure, the kinetic energy begins to decrease. This is called the coasting, or overshoot phase. The maximum bubble is reached when $K_{\text {ext }}$ drops to zero (at $V \sim 4 \times 10^{3} \mathrm{~cm}^{3} / \mathrm{g}$ ). After this time the bubble collapses.

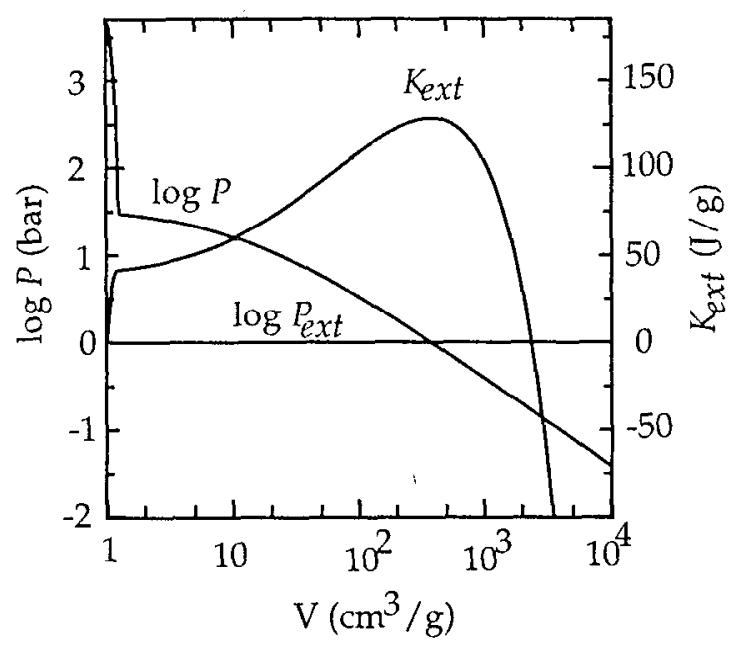

Figure 3. Variation of pressure and kinetic energy with specific volume.

The curves follow an adiabatic expansion. The maximum bubble size is reached when the kinetic energy drops to zero, at $\mathrm{V}=4 \times 10^{4}$

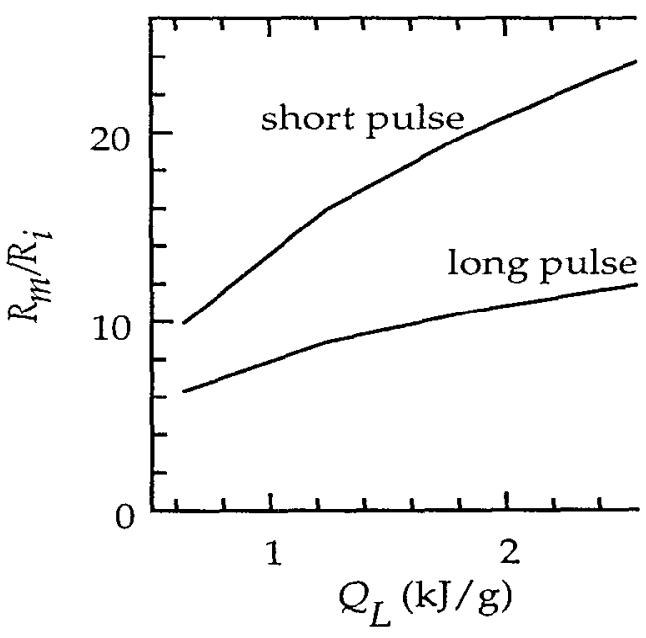

Figure 4. The maximum radius grows with specific laser energy deposition. Short pulses produce larger bubbles than long pulses. All cases have $\mathrm{P}_{\text {ext }}=1$ bar.

In the short pulse limit we can also find the time dependent bubble trajectory and therefrom, the time of maximum size and the equilibrium time. We follow an analysis of the Rayleigh model extended to include an internal bubble pressure 7 . We first derive the kinetic energy of the external fluid to use in Equation (3). For an incompressible spherical flow in an infinite medium, the fluid velocity decreases as the inverse square of the radius:

$$
u=\left(\frac{R}{r}\right)^{2} \frac{\mathrm{d} R}{\mathrm{~d} t}
$$

where $r$ is the radial coordinate of a point external to the bubble, and $\mathrm{d} R / \mathrm{d} t$ is the velocity of the bubble boundary. The kinetic energy is found by integrating: 


$$
K_{e x t}=\frac{1}{2} \rho_{e x t} \int_{R}^{\infty} u^{2} d V=2 \pi \rho_{e x t}\left(\frac{\mathrm{d} R}{\mathrm{~d} t}\right)^{2} R
$$

The net work done by the bubble on the external fluid, minus that done by the stationary fluid far from the bubble is:

$$
W=\int\left(P-P_{\text {ext }}\right) d V .
$$

Equating the kinetic energy to the work and we find the following integral expression relating the bubble radius and time:

$$
t=\sqrt{2 \pi \rho} \int_{R_{o}}^{R}\left(\frac{R^{2}}{W}\right)^{1 / 2} d R
$$

Equation 11 can be evaluated a various radii to give the whole bubble trajectory during the expansion phase. The EOS is used along with the adiabatic condition relating $\mathrm{P}(\mathrm{V})$.

\section{RESULTS}

In Figure 4, we show the maximum bubble radius versus specific laser energy for the long pulse and short pulse cases, using Eqs. (4) and (7) respectively. In both cases the radii grow increasing laser energy. The radii for short pulse are about $x x$ times those for long pulse. This is due to the overshoot of the equilibrium radius which occurs in the short pulse.

For short pulses, we can also find the radius versus time, using Eq. (11). Results are shown in Figure 5, for three values of $Q_{L}$. The time is expressed in terms of a characteristic timescale, (essentially the Rayleigh time for the initial radius)

$$
t_{o}=\sqrt{\frac{\rho_{\text {ext }}}{P_{\text {ext }}}} R_{i}=10 \mu \mathrm{s}\left(\frac{R_{i}}{100 \mu \mathrm{m}}\right)\left(\frac{P_{\text {ext }}}{1 b a r}\right)^{-1 / 2} .
$$

The equilibrium time $t_{e q}$ is found from the trajectory for the short pulse case, and shown in Figure 6. For all cases it is approximately equal to the characteristic Rayleigh time for the initial heated volume, as given by Eq. (12).

In Figure 7, we show the model results $\left(R_{m}, t_{m}\right.$ and $\left.t_{e q}\right)$ in dimensional units, choosing a typical value of $R_{i}(50 \mu \mathrm{m})$ and for a pr.

In Figure 8, we show bubble radii calculated with the LATIS ${ }^{8}$ hydrodynamic simulation code compared to results of the scaling model in the short and long pulse limits compared to bubble. We see good agreement in both limits. In the short pulse limit the scaling model overpredicts the maximum bubble radius by $15 \%$, probably due to its omission of acoustic radiation, which is included in the hydro model. In the long pulse limit, the hydro simulation oscillates about the scaling model prediction, as expected. Such comparisons give us confidence in the scaling models results for approximate estimates of the bubble radius for a wide range of parameters.

In summary, we have presented a scaling model which provide quick estimates of bubble dynamics. It is based on 1-D spherical geometry. It uses thermodynamic and kinetic energy conservation equations and a realistic equation-of-state. With this model we have identified the short and long pulse limits of bubble dynamics. We have been able to predict the bubble 
radius and duration for a wide range of input parameters. This model is useful for prelimary designs of laser produced bubble experiments for a wide range of applications.

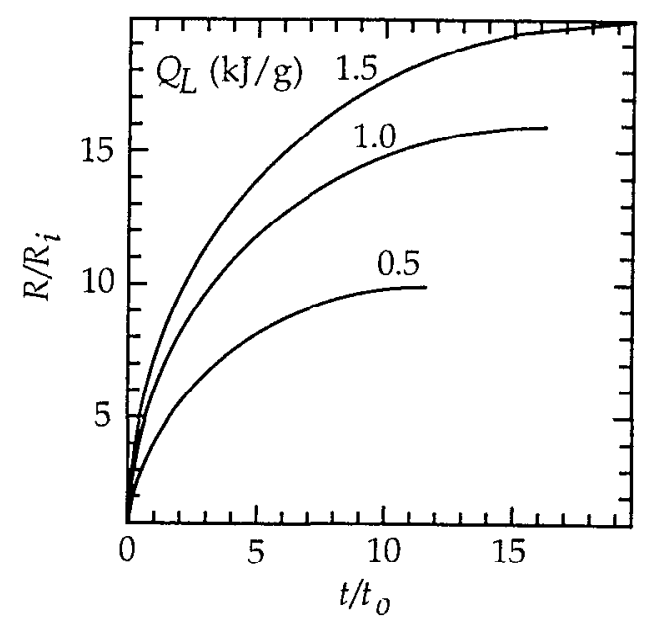

Figure 5. The bubble trajectory up until the time of maximum radius is shown versus time for three specific energy deposition values. All cases are for short pulses and $P_{\text {ext }}=1$ bar.

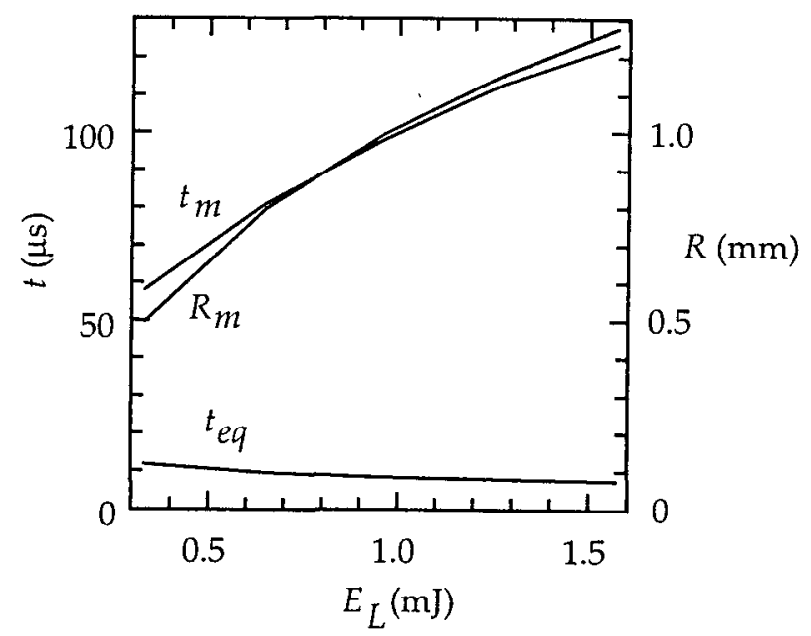

Figure 7. Maximum bubble radius, time of maximum and equilibrium time versus laser energy for a $50 \mu \mathrm{m}$ radius deposition region.

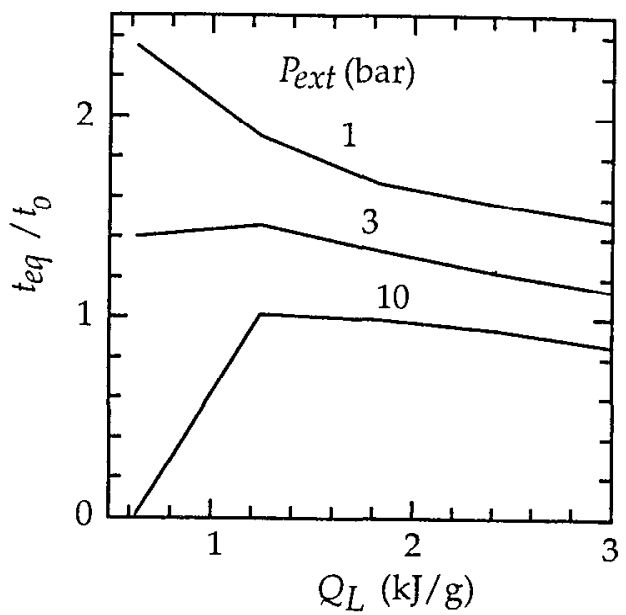

Figure 6. The pressure equilibration timescale is shown versus specific energy deposition for three values of the external pressure.

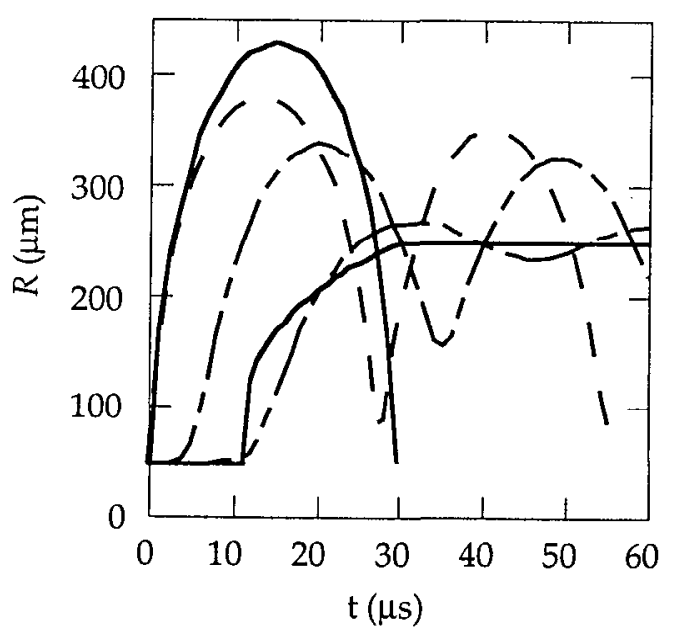

Figure 8. The scaling model compares well with numerical simulations. The dashed lines are LATIS results for laser pulses of 1 ns, $10 \mu \mathrm{s}$ and $30 \mu \mathrm{s}$. The solid lines are the short and long pulse scaling model results 


\section{ACKNOWLEDGMENTS}

This work was performed under the auspices of the U.S. Department of Energy by the Lawrence Livermore National Laboratory under Contract No. W-7405-Eng $\bullet 48$.

\section{REFERENCES}

1. L. Rayleigh, "On the pressure developed in a liquid during the collapse of a spherical cavity," Philos. Mag., 34, p. 94-98, 1917.

2. J. G. Kirkwood and H. A. Bethe, "The pressure wave produced by an underwater explosion, I," OSRD Report No. 588, 1942. M. S. Plesset, "The dynamic of cavitation bubbles," J. Appl Mech., 16, p. 278-231, 1949.

3. M. E. Glinsky, P. A. Amendt, D. S. Bailey, R. A. London, and M. Strauss, "Extended Rayleigh model of bubble evolution with material strength compared to detailed dynamic simulations," in Laser-Tissue Interaction VIII, Ed.: S. L. Jacques, Proc. SPIE 2975, p. 318-334, (SPIE, Bellingham, 1997).

4. M. Strauss, P. Amendt, R. A. London, D. J. Maitland, M. E. Glinsky, P. Celliers, D. S. Bailey, and D. A. Young, "Computational modeling of laser thrombolysis for stroke treatment," in Lasers in Surgery VI, Ed.: R. Anderson, Proc. SPIE 2671, p. 11-21, (SPIE, Bellingham, 1996). P. Amendt, M. Strauss, R. A. London, M. E. Glinsky, D. J. Maitland, P. M. Celliers, S. R. Visuri, D. S. Bailcy, D. A. Young, and D. Ho, "Modeling of bubble dynamics in relation to medical applications," in Laser-Tissue Interaction VIII, Ed.: S. L. Jacques, SPIE 2975, p. 362373, (SPIE, Bellingham, 1997). E. J. Chapyak, and R. P. Godwin, "A comparison of numerical simulations and laboratory studies of laser thrombolysis," in Lasers in Surgery VII, Ed.: R. Anderson, Proc. SPIE 2970, p. 28-34, (SPIE, Bellingham, 1997).

5. A. Vogel, K. Nahen, J. Noack, S. Busch, U. Parlitz, and R. Birngruber, "Energy balance of optical breakdown in water," in in Laser-Tissue Interaction IX, Ed.: S. L. Jacques, Proc. SPIE 3254, in press (SPIE, Bellingham, 1998).

6. L. Haar, J. S. Gallagher, and G. S. Kell, NBS/NRC Steam Tables, (McGraw-Hill, NY, 1984). An updated version by S. Klein and A. Harvey is available from NIST at: srdata@nist.gov.

7. R. T. Knapp, J. W. Daily, and F. G. Hammitt, Cavitation, Chap. 4, (McGraw Hill, New York, 1966).

8. R. A. London, M. E. Glinsky, G. B. Zimmerman, D. S. Bailey, D. C. Eder, and S. I. Jacques, "Laser-Tissue Interaction Modeling with LATIS," App. Optics, 36, 9068-9074, 1997. 


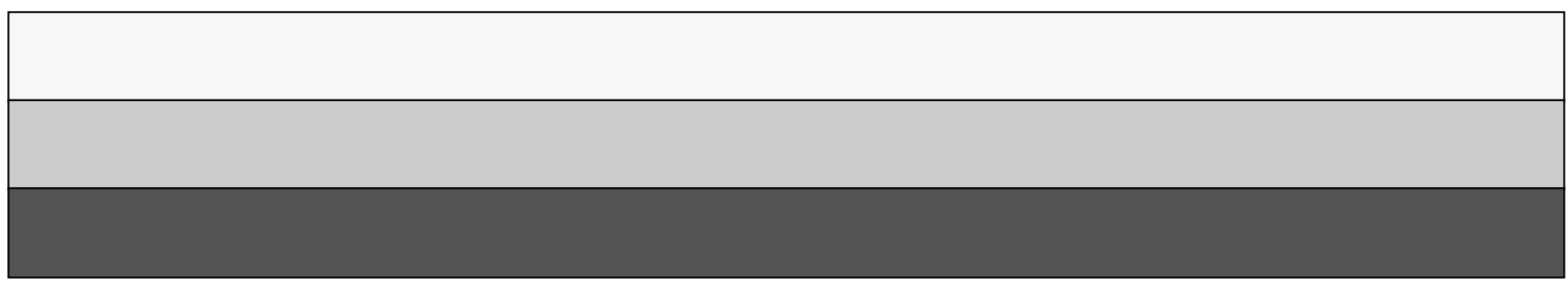

